\title{
Interferometry for the LISA technology package (LTP) aboard SMART-2
}

\author{
G Heinzel $^{1}$, C Braxmaier ${ }^{2}$, R Schilling ${ }^{1}$, A Rüdiger ${ }^{1}$, D Robertson ${ }^{3}$, \\ M te Plate ${ }^{4}$, V Wand $^{1}$, K Arai ${ }^{5}$, U Johann ${ }^{2}$ and K Danzmann ${ }^{1}$ \\ ${ }^{1}$ Max-Planck-Institut für Gravitationsphysik (Albert-Einstein-Institut), Callinstrasse 38, \\ D-30167 Hannover, Germany \\ 2 Astrium GmbH, 88039 Friedrichshafen, Germany \\ 3 Department of Physics and Astronomy, University of Glasgow, UK \\ ${ }^{4}$ ESTEC, Noordwijk, The Netherlands \\ ${ }^{5}$ National Astronomical Observatory of Japan, Mitaka, Tokyo, Japan \\ E-mail: gerhard.heinzel@aei.mpg.de
}

Received 7 November 2002, in final form 14 February 2003

Published 28 April 2003

Online at stacks.iop.org/CQG/20/S153

\begin{abstract}
The interferometer of the LISA technology package (LTP) on SMART-2 is needed to verify the performance of the gravitational sensors by monitoring the distance between two test masses with a noise level of $10 \mathrm{pm} \mathrm{Hz}^{-1 / 2}$ between $3 \mathrm{mHz}$ and $30 \mathrm{mHz}$. It must continuously track the motion of the test mass distance while that distance changes by many $\mu \mathrm{m}$ with a speed of up to $20 \mu \mathrm{m} \mathrm{s}^{-1}$, without losing track of the sign of the motion and without exerting any influence on the test masses that might lead to a motion above that level. As a result of a detailed comparison study, a heterodyne MachZehnder interferometer was selected as the baseline for the SMART-2 mission. Its design and expected performance are described in this paper.
\end{abstract}

PACS numbers: 07.60.Ly, 95.55.Ym, 95.75.Kk

(Some figures in this article are in colour only in the electronic version)

\section{Introduction}

LISA [1] relies on several technologies that have never been tested and cannot be tested on Earth:

- Gravitational sensors: These consist of a test mass in a cage with capacitive sensors/actuators. The test mass is freely floating, subject only to gravitational forces, and the spacecraft shields and follows it.

- Low-noise micronewton thrusters: These compensate all non-gravitational forces acting on the spacecraft. 
- Ultra-stable interferometry in space: In LISA, interferometry is a separate, very difficult problem. In SMART-2, the interferometer is a diagnostic tool for the gravitational sensors with very different requirements. Nevertheless some key components of precision interferometry in space must be used and can be tested:

- stabilized lasers,

- fibre coupling,

- modulators,

- beamsplitters and other optical components,

- ultra-stable optical bench including mounting and alignment techniques,

- photodiodes,

- phase readout of interferometric signals, etc.

These items will be included in the LISA technology package (LTP) [2], one of the scientific payloads on SMART-2. SMART stands for 'Small Missions for Advanced Research in Technology' in ESA's scientific programme. SMART-2 is the second in this 'family' of missions, and is to be launched in 2006.

\section{Requirements}

The main purpose of the LTP interferometer is to verify the performance of the gravitational sensors by monitoring the distance between two test masses, which are approximately $30 \mathrm{~cm}$ apart. For the interferometer a space of approximately $200 \times 200 \mathrm{~mm}^{2}$, in the form of an optical bench (a slab of ULE or Zerodur), centred between the two test masses will be available. Optical components will be mounted by hydroxide catalysis bonding on the baseplate, which will be in a thermally very stable environment.

There are several possible modes of operation, e.g., the spacecraft follows one of the test masses and the other one is left freely floating; the second test mass may be controlled in only some of its degrees of freedom, or the spacecraft follows a linear combination of both test mass positions with other linear combinations left freely floating, etc.

The requirements of SMART-2 have been relaxed by a factor of ten both in frequency and sensitivity as compared to LISA (see figure 1). The interferometric sensing must be able to monitor the test mass position along the sensitive axis (called the $x$-axis) with a displacement noise level of $10 \mathrm{pm} \mathrm{Hz}^{-1 / 2}$ between $3 \mathrm{mHz}$ and $30 \mathrm{mHz}$, relaxing as $1 / f^{2}$ towards $1 \mathrm{mHz}$. The interferometer must do so without exerting any influence on the test masses that might lead to a motion above that level. Furthermore, it must track the motion of the test mass distance while that distance changes by many $\mu \mathrm{m}$ with a speed of up to $20 \mu \mathrm{m} \mathrm{s}^{-1}$, without losing track of the sign of the motion.

The interferometer noise budget is chosen a factor of 8.5 below that SMART-2 mission goal. Each individual noise contribution is allocated another factor of 10 below that (see figure 2). This conservative requirement takes into account the possibility that some noise sources are correlated and might add linearly instead of quadratically.

The minimal measurement required is the distance between test masses, $\Delta x=x_{1}-x_{2}$. Additional optional measurements that were considered during the interferometer selection process are: the distances between each individual test mass and the optical bench, $x_{1}$ and $x_{2}$; the relative positions of the test masses in the $y$-direction, $y_{1}, y_{2}$ and $\Delta y$; and the angular alignment of the test masses. Note that no absolute length will be measured with high precision, but only fluctuations within the measurement frequency band.

The dynamic range of the interferometer must be at least $100 \mu \mathrm{m}$, i.e., many wavelengths of $1064 \mathrm{~nm}$ light. That makes it difficult to use traditional interferometers (Fabry-Perot 

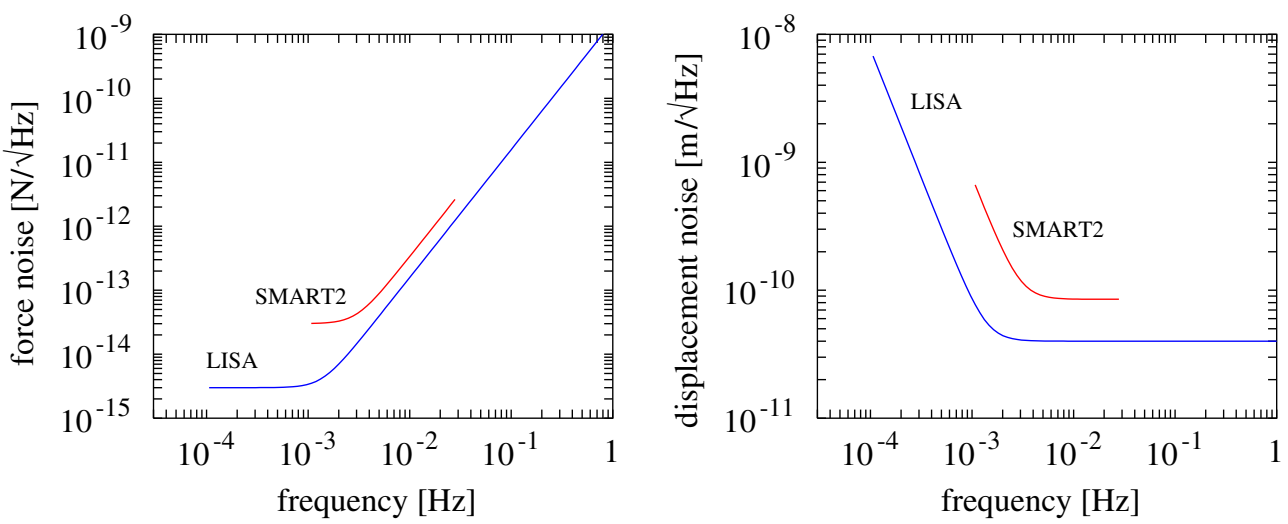

Figure 1. Noise budget for LISA and SMART-2.

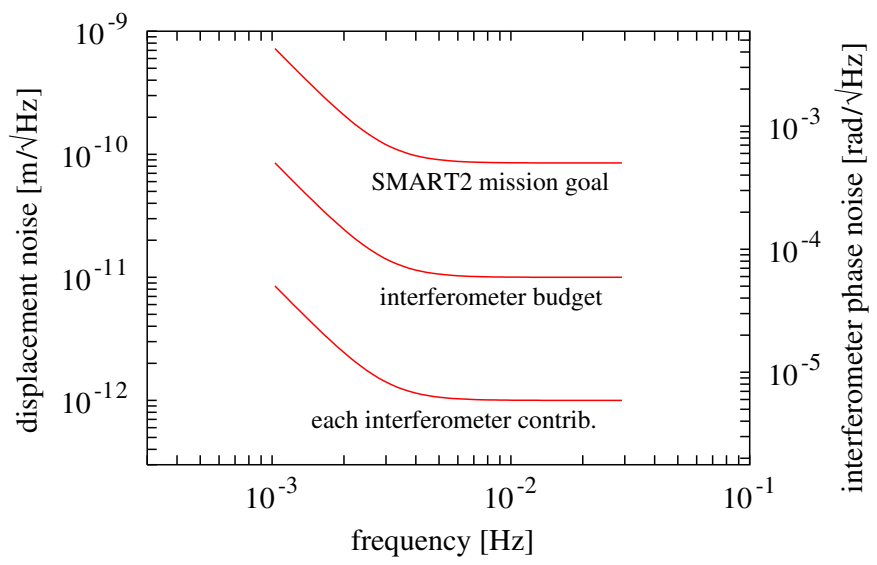

Figure 2. Noise budget for the SMART-2 interferometer.

cavities, Michelson on dark fringe) because these require an actuator to keep the interferometer at a specified operating point. No actuator with the required linearity and dynamic range is currently available. Large-scale mechanical motion is not allowed because of its effect on the test masses. Hence we need an interferometer that yields a constant signal at any operating point. Furthermore, it must be able to track a motion with arbitrary changes of direction.

\section{Interferometer comparison and selection}

During the first months of 2002, Astrium Immenstaadt and AEI Hannover conducted a detailed interferometer comparison study. It quickly became clear that the baseline will be a heterodyne Mach-Zehnder interferometer.

The principle of operation is illustrated in figure 3. The laser beam is split into two parts that are separately frequency-shifted by one acousto-optical modulator (AOM) for each beam. The two AOM driving frequencies differ by a constant amount, the heterodyne frequency $f_{\text {het }}$, which is of the order of $1 \mathrm{kHz}$. After travelling different paths, the two beams are made to interfere at a beamsplitter. The resulting photocurrent in a photodiode contains a strong 

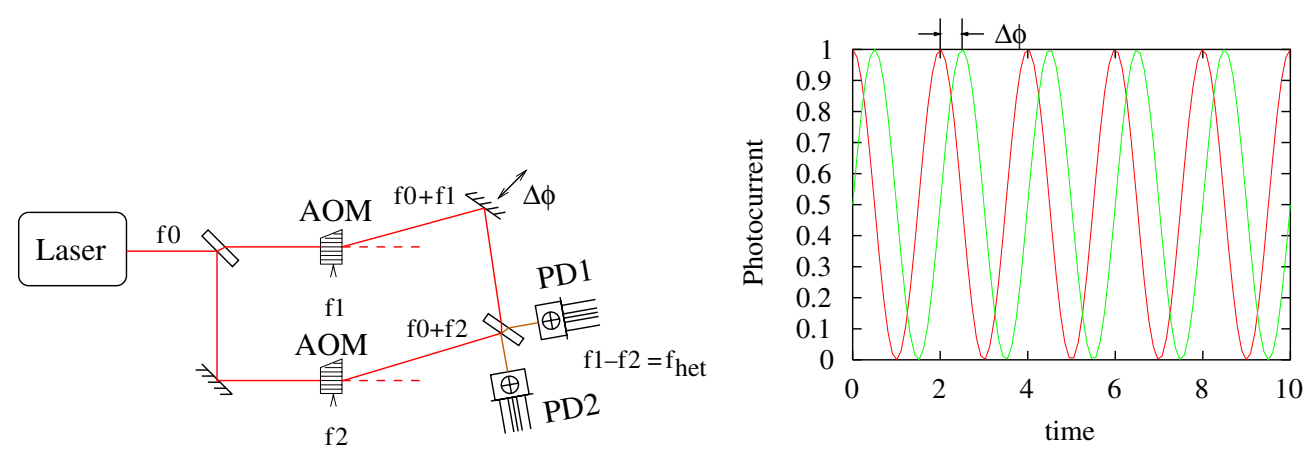

Figure 3. Heterodyne Mach-Zehnder interferometer: schematic (left) and typical photodiode signal (right).

component at the heterodyne frequency $f_{\text {het }}$. The time dependence of the heterodyne signal is given by

$$
\cos \left(2 \pi f_{\text {het }} t-\frac{2 \pi l}{\lambda}\right)=\cos \left(2 \pi f_{\text {het }} t-\varphi\right),
$$

where $l$ is the pathlength difference and $\lambda=1.064 \mu \mathrm{m}$, the laser wavelength. Any differential pathlength variation $\delta l$ hence translates into a phase variation $\delta \varphi$ of that heterodyne signal, which will be measured electronically with respect to a phase reference derived from another auxiliary interferometer that is not subject to the pathlengths variations. Thus all phase-shifting effects that occur in the generation of the two beams (e.g., AOM drivers, fibre expansion, etc) are completely suppressed in the phase measurement.

In designing an interferometer that directly measures the distance between the test masses, $x_{1}-x_{2}$, the problem arises how to separate incoming and outgoing beams on the axis that connects the test masses.

Many interferometers that were investigated during the comparision study use polarizing components, which are known to have a number of problems, in particular when the temperature changes. It is very hard to find relevant data about polarizing components in the literature or from manufacturers. A quick experiment in our own lab mainly showed the difficulty in obtaining reproducible results and the necessity for fine-tuning all alignment degrees of freedom, which will be impossible for SMART-2.

Although we cannot prove that polarizing components will spoil the interferometer performance, we cannot guarantee to reach the performance, either. Hence we decided to use a non-polarizing interferometer. The price we have to pay is to give up the normal incidence on the test masses and compactness of the design.

\section{Baseline design}

The result of the interferometer comparison study is the present baseline design, a nonpolarizing heterodyne Mach-Zehnder interferometer described in detail below. It measures

- the distance between the two test masses (called $x_{1}-x_{2}$ ),

- the position of one test mass with respect to the optical bench (called $x_{1}$ ),

- the differential alignment of both test masses with respect to the optical bench, as it appears in the angular fluctuation of the reflected beams,

- the alignment of one test mass with respect to the optical bench. 


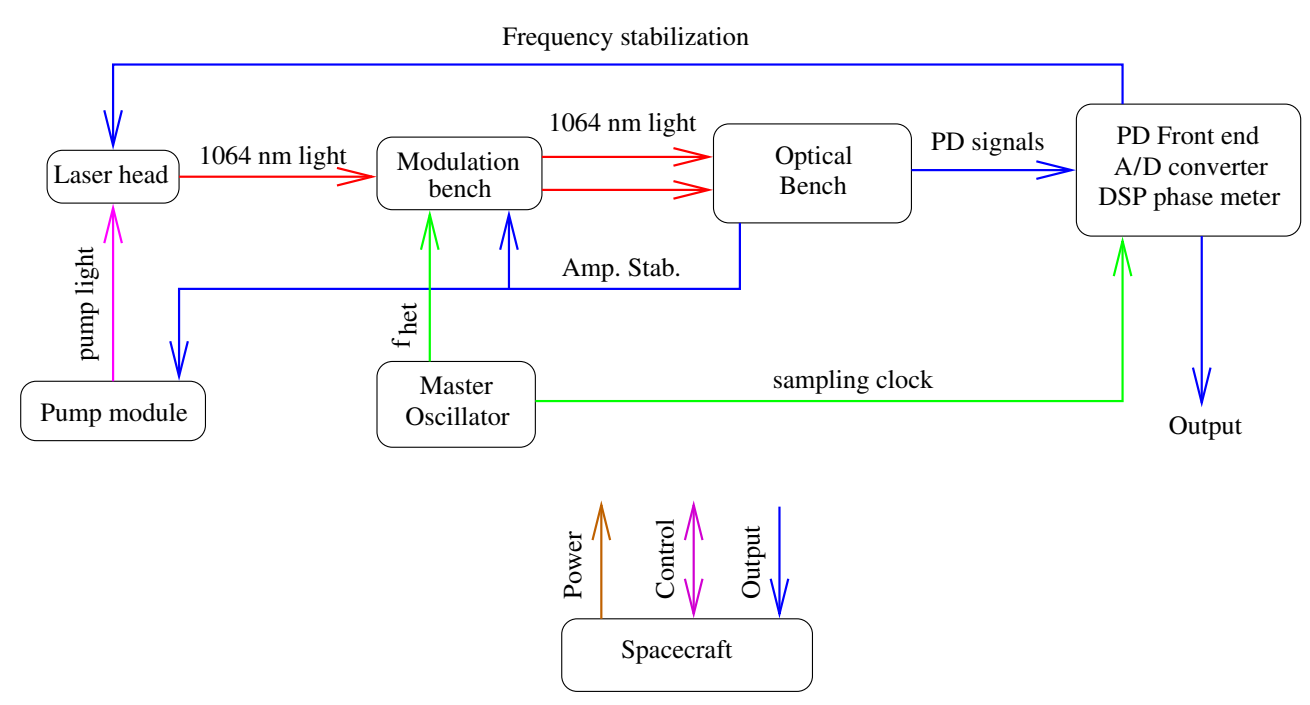

Figure 4. Overview of the SMART-2 interferometer.

We use a Nd:YAG laser (1064 nm) with a power of approximately $25 \mathrm{~mW}$ arriving at the end of an optical fibre. Its power and frequency will both be stabilized, as discussed below. Apart from the fibre-coupled laser source, the interferometer contains two separate items: the modulation bench and the optical bench. Figure 4 shows an overview of the main subsystems comprising the interferometer.

On the modulation bench, the two frequency-shifted laser beams are prepared as described in section 3. Both the input (from the laser) and the two outputs (to the optical bench) are fibre-coupled. The reason for a separate modulation bench is to avoid heat dissipation on the optical bench. A sketch of the optical bench is shown in figure 5. It contains four separate interferometers:

- $x_{1}-x_{2}$ : This interferometer provides the main measurement: the distance between the two test masses and their differential alignment.

- $x_{1}$ : This interferometer provides as auxiliary measurement the distance between one test mass and the optical bench and the alignment of that test mass.

- Reference: This interferometer provides the reference phase for the above two measurements.

- Frequency: This interferometer uses basically the same interfering beams as the 'reference' interferometer but with intentionally unequal pathlengths so as to measure laser frequency fluctuations.

The two frequency-shifted beams, of approximately 5-8 $\mathrm{mW}$ each, that arrive on the optical bench via fibres from the modulation bench are drawn in red (solid) and blue (dashed), respectively.

Fractions of approximately $1 \mathrm{~mW}$ from each beam are sampled by pickoff mirrors (beamsplitters), and directed onto photodiodes for the power stabilization. The signals will be split into a common mode part and a differential part which will be fed back to the laser and the AOM drivers, respectively.

A further set of beamsplitters (three each for the red and blue beams) split each beam into four beams of equal intensity. The four red beams are the measurement beams of the 


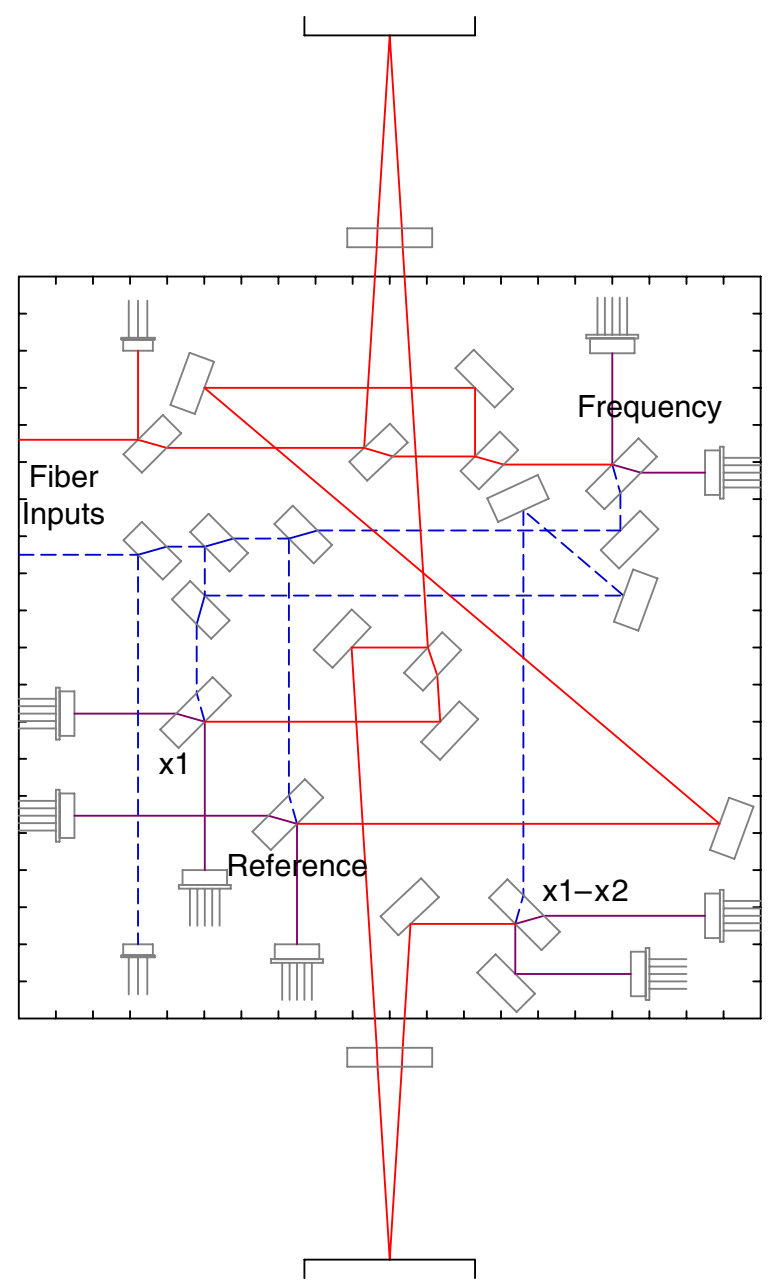

Figure 5. Sketch of the optical bench in the baseline design. Drawing is to scale, with the outer frame representing the $200 \times 200 \mathrm{~mm}$ baseplate area.

four interferometers, while the corresponding four blue beams act as reference beams. In each interferometer the number of transmissions and reflections is equal for the red and blue beams being used.

In each interferometer, the red and blue beams are recombined at a final beamsplitter. The two complementary output beams are directed onto a quadrant photodiode each. Due to the frequency difference between the red and blue beams, a beat note at the heterodyne frequency will appear on each photodiode. The phase of this beat note depends on the pathlength difference between the red and blue beam paths. Pathlength differences induced by the modulation bench, AOMs, fibres and power stabilization beamsplitters are eliminated by using the beat note from the 'reference' interferometer as phase reference.

The paths travelled by the reference and measurement beams are as similar as possible to reduce the effects of laser frequency noise. If an extra optical pathlength of approximately $40 \mathrm{~cm}$ is included in the blue light path (e.g., in the fibre) before that light enters the optical 


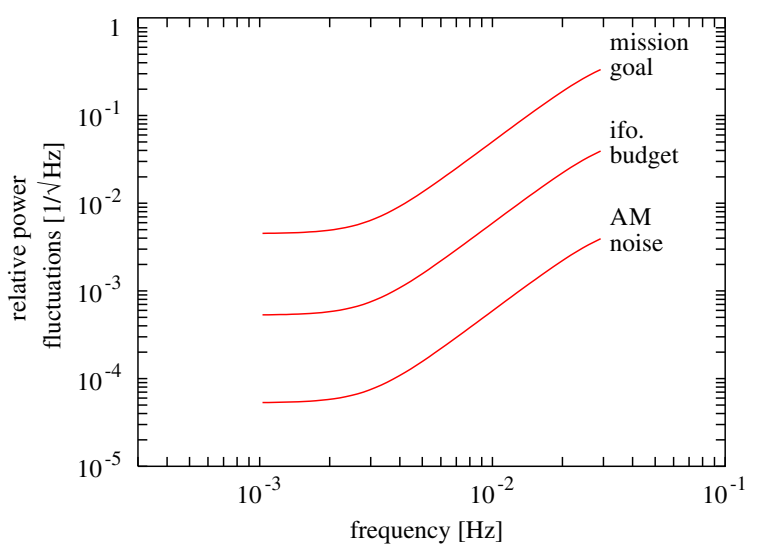

Figure 6. Power stability requirements. The three curves represent the mission noise budget, total interferometer budget and budget for each interferometer noise contribution, expressed as equivalent laser power fluctuations. The lowest curve is the actual laser power stability requirement.

bench, the red and blue pathlength differences can be made virtually equal for the three interferometers labelled ' $x_{1}$ ', ' $x_{1}-x_{2}$ ' and 'reference'.

The last interferometer labelled 'frequency' interferes beams that have travelled intentionally unequal pathlengths and is thus sensitive to frequency fluctuations of the laser source. The conversion factor of frequency fluctuations $\delta \omega\left(\mathrm{rad} \mathrm{s}^{-1}\right)$ to phase fluctuations $\delta \varphi$ $(\mathrm{rad})$ is given by the differential time delay $\Delta l / c$. The output signal from this interferometer can be used to stabilize the frequency of the laser and/or to correct the main output signal.

The optical layout of the interferometer was entered into OPTOCAD (a Gaussian beam raytracing software developed by one of the authors (RS) for the ground-based interferometers). Optocad computes all beams, including stray beams. The results show that at least some stray beams must be suppressed, e.g., by selective coating or blackening of beamsplitters.

\section{Power stabilization}

The laser power $P$ reflected from the gravitational sensors produces a force on the masses. Fluctuations in the laser power will thus produce fluctuating forces on the gravitational sensors which could limit the sensitivity of the measurement. The displacement fluctuation $\delta x$ caused by the power fluctuation $\delta P$ is given by

$$
\delta x=\frac{2 \delta P}{m c \omega^{2}},
$$

where $m$ is the test mass (assumed to be $1 \mathrm{~kg}$ ) and $\omega$ the Fourier frequency of the fluctuation. Accordingly, the required relative power stability for $1 \mathrm{~mW}$ of light in the measurement arm is found (assuming an allowed $\widetilde{\delta x}$ of $1 \mathrm{pm} \mathrm{Hz}^{-1 / 2}$ and $f=3 \mathrm{mHz}$ ) by

$$
\frac{\widetilde{\delta P}}{P}=\frac{m c \omega^{2}}{2 P} \tilde{\delta x} \approx 5 \times 10^{-5} \mathrm{~Hz}^{-1 / 2}
$$

between $1 \mathrm{mHz}$ and $3 \mathrm{mHz}$, relaxing as $f^{2}$ for frequencies up to $30 \mathrm{mHz}$ (see figure 6). Note that there is a very conservative total safety factor of 85 (between this noise contribution and the mission goal sensitivity) included in this requirement. 


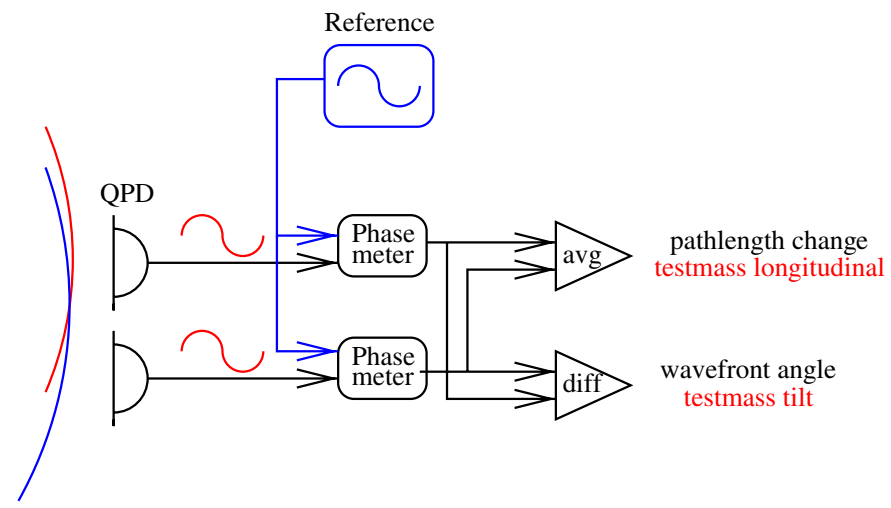

Figure 7. Differential wavefront sensing (only one of the two directions $x$ and $y$ is shown).

At the heterodyne frequency $f_{\text {het }}$, of some $\mathrm{kHz}$, the (more stringent) requirement is $\widetilde{P P} / P \leqslant 10^{-6} \mathrm{~Hz}^{-1 / 2}$ due to direct coupling to the phase measurement. This is still far above the shot noise-limited stability achievable using $1 \mathrm{~mW}$ of light.

In any case, stabilization of the laser power will be necessary. In order to compensate fluctuations of the fibre coupling efficiency and fibre transmission, it will be done by measuring the power at the end of each fibre and feeding a correction signal back to the respective AOM driver.

\section{Alignment measurements}

If each photodiode were a single-element photodiode, only the longitudinal information (motion along $x$-axis) could be determined. More information can be obtained by replacing each photodiode with a quadrant diode.

The sum of all quadrants is used as before for the longitudinal readout. The signals from the individual quadrants are used to determine alignment drifts of the interferometer and, in particular, the test masses by applying the same phase measurement as is done for the longitudinal signal ('differential wavefront sensing'; see figure 7). For large misalignments when the interferometer contrast is too small to obtain useful signals from that phase measurement, the dc output from the quadrants can be used to produce rough alignment information.

The conversion factor of test-mass angle $\alpha$ to (differential) phase readout $\varphi$ is approximately $\varphi / \alpha \approx 5 \times 10^{3}$. Hence the longitudinal sensitivity of $6 \times 10^{-5} \mathrm{rad} \mathrm{Hz}^{-1 / 2}$ for the whole interferometer corresponds to a phase sensitivity of $\widetilde{\delta \alpha} \approx 10^{-8} \mathrm{rad} \mathrm{Hz}^{-1 / 2}$. The actual angular sensitivity may, however, be even better due to common mode rejection of several noise sources. This is expected to be better than the angular readout capability of the capacitive sensors. Indeed this optical alignment readout may be used to stabilize the alignment of the test masses.

\section{Prototype experiment}

A table-top laboratory prototype of the interferometer was built and characterized in Hannover. It consists of two similar interferometers (instead of four), the phases of which are compared. 
Preliminary results are very encouraging and in reasonable accordance with theory. Further results will be published elsewhere.

\section{References}

[1] Astrium et al 2000 LISA: Study of the Laser Interferometer Space Antenna, Final Technical Report ESTEC Contract No 13631/99/NL/MS, Report No LI-RP-DS-009

[2] Vitale S et al 2002 LISA and its in-flight test precursor SMART-2 Nucl. Phys. B (Proc. Suppl.) 110 209-16 